\title{
On Providing End-To-End QoS Introducing a Set of Network Services in Large-Scale IP Networks
}

\author{
E. Tsolakou, E. Nikolouzou, and S. Venieris \\ National Technical University of Athens \\ School of Electrical and Computer Engineering \\ Telecommunications Laboratory \\ 9 Heroon Polytechniou Str, 15773 Athens, Greece \\ \{evi, enik\}@telecom.ntua.gr, ivenieri@cc.ece.ntua.gr
}

\begin{abstract}
The Differentiated Services (DiffServ) architecture has been proposed as a scalable solution for providing service differentiation among flows. Towards the enhancement of this architecture, new mechanisms for admission control and a new set of network services are proposed in this paper. Each network service is appropriate for a specific type of traffic and is realized through its own network mechanisms, which are the Traffic Classes. Traffic Classes provide the traffic handling mechanisms for each Network Service and are composed of a set of admission control rules, a set of traffic conditioning rules and a per-hop behavior (PHB). Different traffic-handling mechanisms are proposed for each network service and are implemented with the use of the OPNET simulation tool. A large-scale network is used as a reference topology for studying the performance and effectiveness of the proposed services.
\end{abstract}

Keywords: Network Services, QoS, Traffic Classes

\section{Introduction}

Motivated by the rapid change of QoS requirements of the new introduced network applications, the Internet has been evolving towards providing a wide variety of services, in order to meet the qualities of information delivery demanded by the applications. For the past few years, there have been two major efforts focusing on augmenting the single-class, best effort Internet to include different levels of guarantee in quality of service - Integrated service (Intserv) and Differentiated service (DiffServ) [1]. The most salient point between these two approaches is the difference on the treatment of packet streams. Intserv tends to emulate circuit-switch networks, focusing on guaranteeing QoS on individual packet flows between communication end-points. To ensure the level of guarantee on a per-flow basis, it requires explicit signaling to reserve corresponding resources along the path between these end-points. One major dilemma faced by this approach is that in the core of the Internet, where exist several millions of flows, it may not be feasible to maintain and control the forwarding states efficiently. These scalability and management problems are addressed recently by DiffServ approach. 
The focal point of the DiffServ model lies in the differentiation of flows at an edge router of a DS-domain and the aggregation of those flows of the same service class at a core router of the DS-domain. At each ingress interface of a edge router, packets are classified and marked into different classes, using Differentiated Services CodePoint (DSCP). Complex traffic conditioning mechanisms such as classification, marking, shaping, and policing are pushed to network edge routers. Therefore, the functionalities of the core routers are relatively simple - they classify packets and then forward them using corresponding Per-Hop Behaviors (PHBs). In this sense, PHB is a means by which a node allocates resources to behavior aggregates, and it is on top of this basic hop-by-hop resource allocation mechanism that useful differentiated services may be constructed. PHBs are implemented in nodes by means of some buffer management and packet scheduling mechanisms and the parameters associated with those mechanisms are closely related to those of traffic conditioning.

\section{Network Services}

In order to provide QoS guarantees in a DiffServ network it is essential to assure QoS differentiation. Therefore, a set of five Network Services (NS) has been specified and implemented in our framework [2], which comprises the services sold by the provider to the potential customers, either end-users or other providers. The specified NSs are: Premium Constant Bit Rate, Premium Variable Bit Rate, Premium Multimedia, Premium Mission Critical and Standard Best Effort.

The PCBR network service is intended to support applications that require VLLlike services, i.e. voice flows, voice trunks, interactive multimedia applications with low bandwidth requirements. That kind of flows is usually characterized by an almost constant bit rate (CBR) and low bandwidth requirements, while a great number of them are unresponsive (UDP). In addition, they should have small packets ( $<256$ Bytes), so as not to provoke long transmission delays. It requires and expects to receive low delay, very low jitter and very low packet loss. The targeted quantitative value for end-to-end delay is less than $150 \mathrm{msec}$ for $99.99 \%$ of the packets, while packet loss is expected to be less than $10^{-6}$.

The PVBR network service mainly copes with unresponsive variable bit rate (VBR) sources. Typical candidate applications are real time video and teleconferencing. The requirements are similar to the PCBR network services but with a less strict needs concerning the jitter and packet loss. They are characterized by large packet size, which oscillates from 256 to 1024 bytes. The targeted end-to-end delay is limited to be less than $250 \mathrm{msec}$ for $99.99 \%$ of the packets, while packet loss should be less than $10^{-4}$.

The PMM is expected to carry a mixture of TCP and non-TCP traffic. These flows require a minimum bandwidth, which must be delivered at a high probability. Independently of the transport protocol, flows are expected to implement some kind of congestion control mechanism and their aggressiveness should be similar to the one of TCP, assuming that they are roughly TCP-friendly [3]. This NS is supposed to serve adaptive applications (TCP), like low-quality video, non real time multimedia applications or file transfer (FTP). They require throughput guarantees, which are translated into low packet loss only for "in-profile" packets $\left(\leq 10^{-3}\right)$. 
PMC is targeting to non-greedy adaptive applications that have great sensitivity concerning packet loss. It is thus suitable for transaction-oriented applications and interactive applications such as online games and chat-like applications. The main characteristics are the non-greediness of the flow, the responsive nature (TCP), the low use of bandwidth and the short life of the connection. The most important requirement is very low packet loss only for "in-profile" packets $\left(\leq 10^{-6}\right)$. Nevertheless, low queuing delay is also desired, in order to retain the meaning of interactiveness.

Finally, packets of the STD BE receive no special treatment in the network.

\subsection{Traffic Classes}

The implementation of the Network Services is realized with the use of some network's mechanisms, which are the Traffic Classes (TCLs). A TCL is defined as a composition of a set of admission control rules, a set of traffic conditioning rules (Fig.1) and a per-hop behavior (PHB). In the proposed architecture five TCLs are introduced: TCL1, TCL2, TCL3, TCL4 and TCL5 which correspond to PCBR, PVBR, PMM, PMC and STD BE. Each TCL maintains a separate queue at the router output ports and allocates one or more DSCPs in order to enable differentiation of packets in the core network. A PHB implemented in the output port of a router is realized in the network with the use of scheduling and buffer management algorithms. The scheduling mechanism selected is a combination of the Priority Queuing [4] and Weighted-Fair Queuing [4], which is called PQWFQ (Fig.2). TCL1 has a strict priority over the other TCLs. The rest TCLs are scheduled with the WFQ and each queue is managed by different weight and queuing strategy [5].

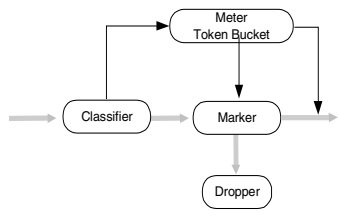

Fig. 1. Traffic Conditioning Mechanisms

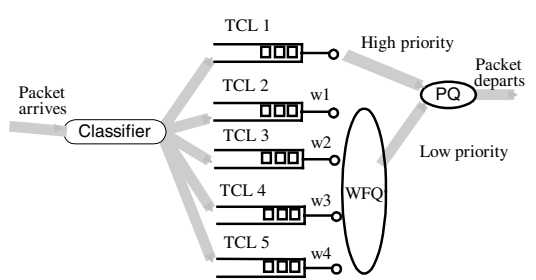

Fig. 2. Design of router output port

According to the WFQ weights, the traffic injected into the network should be limited. Therefore, apart from the traffic classes, specific Admission Control (AC) algorithms should be implemented at the edges of the network to control the admitted number of flows. The proposed AC algorithms for each TCL are described in detail in [6]. Moreover, specific policing actions are deployed to ensure that non-conforming data flows do not affect the QoS requirements for already active data flows. Policing at the network access point is performed through a token bucket (TB) device (r,b). A specific traffic profile is determined for each NS, which best characterizes the data source. 


\section{Simulations}

The simulations were realized in a large-scale network topology. This topology consists of five interconnected networks, which belong to five cities of Europe. Three of them are considered as transit networks, which are situated in Munich, Vienna and Rome. The traffic generators are placed in the network of Athens and the destination network is London for all TCLs, in order to choose the longest path. The routers compromising the end-to-end topology are depicted in Fig.3. Background generators are placed in different links and different domains, rising five different bottlenecks in the network. The EIGRP is considered as the routing protocol for the whole network. The recommended AC limits for each TCL are configured as: 10\% for TCL1, 15\% for TCL2, 30\% for TCL3, 5\% for TCL4 and for TCL-STD (BE) is dedicated the rest of the link. Regarding the BT, each TCL is considered with the maximum admitted value of traffic.

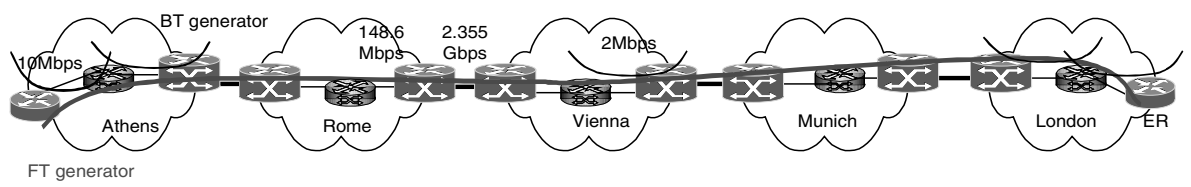

Fig. 3. End-to-End Path

\subsection{Study of Tcl1 \& Tcl2}

TCL1-PCBR is served as foreground traffic using a voice flow. The performance of TCL1 was validated assuming target packet loss ratio to be $10^{-6}$.According to the specified AC, the maximum admissible load is $\rho=0.52$, that is equivalent to $104 \mathrm{kbps}$. Therefore, a single TB [6] for TCL1 was configured with $\mathrm{PR}=104 \mathrm{kbps}$ and $\mathrm{BSP}=256 \mathrm{Bytes}$. The buffers in the routers were set to 5 packets for TCL1. The endto-end delay for different packet sizes was measured without any BT (Fig.4). The end-to-end delay of TCL1 (130Bytes packet size) was also measured for a sequentially increasing number of bottlenecks in the network (Fig.5). The basic conclusion is that increasing the amount of BT the end-to-end delay is being increased up to three times. Although, this value still remains low and less than $150 \mathrm{msec}$.

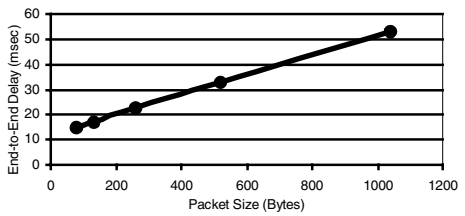

Fig. 4. Av. end-to-end delay vs packet size

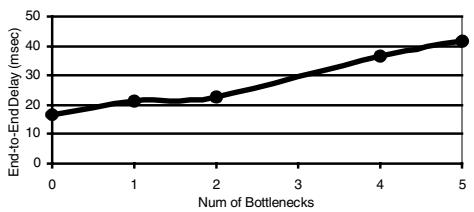

Fig. 5. Av. end-to-end delay vs bottlenecks

TCL2-PVBR class is served as foreground traffic where video flows. Assuming that the $\mathrm{AC}$ limit is $300 \mathrm{kbps}$ and the target packet loss equal to $10^{-4}$, the effective bandwidth for each admitted flow is $34,650 \mathrm{kbps}$, where each flow is characterized by $\mathrm{PR}=32 \mathrm{kbps}, \mathrm{SR}=24 \mathrm{kbps}$ and packet size 400bytes. Therefore, the number of admitted 
flows is 8 . The buffer size in routers for TCL2 was set to 5 packets, in order to avoid long queuing delays. A dual TB [6] was consequently configured for each flow, with $\mathrm{PR}=32 \mathrm{kbps}, \mathrm{BSP}=1000 \mathrm{~B}(2 * \mathrm{M}), \mathrm{SR}=24 \mathrm{kbps}$ and $\mathrm{BSS}=5000 \mathrm{~B}(10 * \mathrm{M})$. The average end-to-end delay for each flow was measured as depicted in Fig.6, where no BT was used. Moreover, the maximum end-to-end delay was measured having different bottlenecks. These results are depicted in Fig.7. As a final result was that increasing the BT injected in the network, the max. observed end-to-end delay is increased of up to two times; though it still remains less than $250 \mathrm{msec}$.

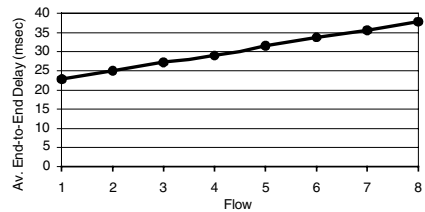

Fig. 6. Av. end-to-end delay vs flow of TCL2

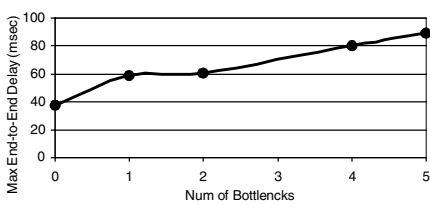

Fig. 7. Max. end-to-end delay vs bottlenecks

\subsection{Study of Tcl3 \& Tcl4}

TCL3-PMM is served as foreground traffic that is targeted for low-quality video and file transfer applications. The dedicated bandwidth for TCL3 was set to $540 \mathrm{kbps}$, where AC limit was $600 \mathrm{kbps}$ and the target utilization factor equal to 0.9. Five TCP flows were used for TCL3 with a mean rate of $108 \mathrm{kbps}$ and packet size equal to 1000Bytes. A single TB [6] was configured for each flow with $\mathrm{SR}=108 \mathrm{kbps}$ and $\mathrm{BSS}=10,000 \mathrm{~B}(10 * \mathrm{M})$. The configuration of the WRED algorithm [3] on a $2 \mathrm{Mbit} / \mathrm{s}$ link is for "out-profile" packets: $\min _{\mathrm{th}}=18, \max _{\mathrm{th}}=38$, and $1 / \operatorname{maxp}=9$, for "in-profile" packets: $\min _{\mathrm{th}}=38, \max _{\mathrm{th}}=97$, and $1 / \mathrm{maxp}=88$. The buffer size was set to 130 packets and the packet loss ratio was considered to be less than $10^{-3}$. The results show, that the capacity $(600 \mathrm{kbps})$ is shared among these five TCP connections in a fair manner. The total throughput is depicted in Fig.8. This throughput is decreased up to the scheduled bandwidth of TCL3, when a BE traffic is occurred. The measured value of packet loss for "in-profile" packets was $3 * 10^{-4}$, when the simulation time was $5 \mathrm{~min}$.

TCL4-PMC is served as foreground traffic. PMC traffic is simulated through ON/OFF sources with constantly distributed ON/OFF times. During ON time the source sends TCP packets with an average rate of $23 \mathrm{kbps}$ for $2 \mathrm{sec}$ with a packet size 500Bytes. The OFF time was set to $2 \mathrm{sec}$. According to the AC limit, the effective bandwidth for each flow is equal to $19.63 \mathrm{kbps}$; so 5 flows will be admitted. A dual TB [6] for TCL4 was configured, with $\mathrm{PR}=32 \mathrm{kbps}, \mathrm{BSP}=1000 \mathrm{~B}(2 * \mathrm{M}), \mathrm{SR}=14 \mathrm{kbps}$ and $\mathrm{BSS}=5,000 \mathrm{~B}\left(10^{*} \mathrm{M}\right)$. A FIFO with two thresholds was considered as the buffer management. The buffer size was set to 35 packets and the dropping threshold for "out-profile" packets to 10 and for "in-profile" packets to 35. The end-to-end delay for TCL4 was measured having different bottlenecks (Fig.9). The measured value of packet loss for the "in-profile" packets was $9 * 10^{-6}$, when the simulation time was $12 \mathrm{~h}$. Consequently, the average end-to-end delay increases while increasing the BT injected in the network, but it still remains low. 


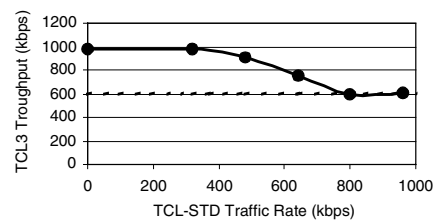

Fig. 8. TCL3 Throughput of five TCP flows

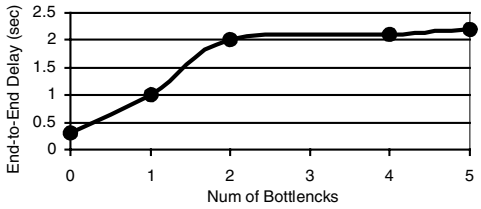

Fig. 9. Average end-to-end delay for TCL4

\section{Conclusions \& Future Work}

The work presented in this paper dealt with the definition and deployment of a set of Network Services within a DiffServ-enabled core network architecture. The Network Services, which are implemented in the network with the traffic handling mechanisms offered by respective Traffic Classes, target at different kinds of user traffic that exhibit similar QoS requirements and characteristics, and they therefore demand analogous treatment within the network. We propose five Network Services that can accommodate most of the well-known application traffic usually submitted in a network. A different set of mechanism is used for each TCL, based on flows characteristics and the corresponding QoS requirements. Subsequently, simulation results proved that the proposed traffic handling mechanisms are adequate for the proposed Network Services, even under the proposed large-scale topology, which compromises a worst-case scenario. Therefore, the correctness of our design was verified, since the target QoS performance was achieved for all the NSs. Future work would focus on refinement of the proposed traffic control mechanisms (traffic conditioner, buffer management, scheduling) and on performance studies using different traffic models.

Acknowledgements. This paper is partly funded by the European research project AQUILA, Information Societies Technology (IST) programme, IST-1999-10077. The authors are currently engaged in the definition, implementation and evaluation of the concept presented in this paper.

\section{References}

[1] Black, D.et.al., "An Architecture for Differentiated Services”, RFC 2475, December 1998.

[2] Deliverable D1201, System architecture and specification for the first trial, AQUILA project consortium, http://www-st.inf.tu-dresden.de/aquila/, June 2000.

[3] M. Allman, et.al., "TCP Congestion Control”, RFC 2581.

[4] M. Markaki, E. Nikolouzou, I. Venieris, "Performance Evaluation of Scheduling Algorithms for the Internet", $8^{\text {th }}$ IFIP Conference on Performance Modeling and Evaluation of ATM \& IP Networks, Ilkley, June 2000.

[5] T. Ziegler, C. Brandauer, S. Fdida, "A quantitative model for parameter setting of RED with TCP traffic", 9th International Workshop on Quality of Service, Karlsruhe, Germany, June 6-8, 2001.

[6] Deliverable D1301, Specification of traffic handling for the first trial, AQUILA project consortium, http://www-st.inf.tu-dresden.de/aquila/, September 2000. 